$1 \mid 2002$

Varia

\title{
Philippe Batifoulier (dir.), Théorie des conventions
}

Paris, Economica, 2001

Thierry Kirat et Gilles Raveaud

\section{(2) OpenEdition \\ Journals}

Édition électronique

URL : http://journals.openedition.org/ei/714

DOI : 10.4000/ei.714

ISSN : 2553-1891

Éditeur

Association Économie et Institutions

Édition imprimée

Date de publication : 1 décembre 2002

ISSN : 1775-2329

Référence électronique

Thierry Kirat et Gilles Raveaud, «Philippe Batifoulier (dir.), Théorie des conventions », Économie et institutions [En ligne], 1 | 2002, mis en ligne le 31 janvier 2013, consulté le 23 septembre 2020. URL http://journals.openedition.org/ei/714 ; DOI : https://doi.org/10.4000/ei.714 


\title{
Compte-rendu de : Philippe Batifoulier (dir.), Théorie des conventions, Paris, Economica, sept. 2001.
}

\author{
Thierry Kirat, CNRS-IDHE (ENS de Cachan) \\ Gilles Raveaud, IDHE (ENS de Cachan)* \\ (décembre 2001)
}

Cet ouvrage est le fruit d'un travail collectif mené au sein du FORUM à l'Université de Paris 10-Nanterre sur l'Economie des conventions. Il réunit huit contributions, introduites par une préface d'Olivier Favereau, par une longue introduction générale rédigée par Philippe Batifoulier et Guillemette de Larquier, et clôturées par une postface de François Eymard-Duvernay. Tous les contributeurs ${ }^{1}$ sont membres du FORUM, à Nanterre.

La finalité de l'ouvrage est de faire le point sur l'analyse économique des conventions et, au delà, de poser des jalons pour une intégration du politique dans ce cadre. La première partie, composée de deux chapitres, présente les "origines " de la théorie des conventions. Elle s'appuie essentiellement sur les travaux de Keynes, Lewis et Schelling, dont elle donne une lecture très informée et complète. Cette base posée, les auteurs indiquent que deux approches théoriques distinctes ont développé la notion de convention. La première est l'approche dite "stratégique" des conventions, qui fait l'objet de la deuxième partie. Celle-ci regroupe trois chapitres présentant cette approche, modélisée par la théorie des jeux, et fondée sur la logique de l'intérêt et la rationalité maximisatrice. Ainsi que le résument Batifoulier et de Larquier, l'approche stratégique conduit à faire de la convention le résultat d'une interaction stratégique, et à la définir comme "une règle de comportement qui permet aux individus de se coordonner ou de coopérer" (p. 20). C'est dans la troisième et dernière partie de l'ouvrage qu'est présentée l'approche dite "interprétative" des conventions, qui regroupe trois chapitres, portant sur 1' " affirmation du programme de recherche " de l'Economie des conventions (Ch. 6), 1 ' "interprétation et le fondement conventionnel des règles " (Ch. 7) et la "dynamique des conventions" (Ch. 8). Dans cette approche, les conventions ne sont pas seulement des règles de comportement,

\footnotetext{
* Nous remercions Laure Bazzoli, Nicolas Farvaque et Evelyne Serverin pour leur relecture attentive de ce texte et leurs utiles suggestions.

1 Outre ceux cités, les autres auteurs sont: Philippe Abecassis ; Olivier Biencourt; Camille Chaserant; Frédéric Gannon; Antoine Rebérioux ; Olivier Thévenon ; Pascal Gabriel ; Jacques Merghiers et Daniel Urrutiaguer.
} 
mais aussi des "modèles d'évaluation qui permettent d'interpréter toutes les règles "; pour les auteurs, admettre cela, c'est déplacer le curseur de l'analyse en amont des actions, en direction des "représentations sur les comportements " (ibid.).

L'objet de cette recension n'est pas de développer les qualités de l'ouvrage et des travaux qui l'ont alimenté, lesquelles ne sauraient être contestées. En nous basant essentiellement sur l'introduction $\mathrm{du}$ livre, sa troisième partie et sa postface, nous visons plutôt à apporter des éléments de discussion critique sur trois questions importantes que cet ouvrage permet de poser : (a) assiste-t-on à une "normalisation" de l'Economie des conventions (et laquelle) ? (b) quelle est la portée, en termes de compréhension du fonctionnement des règles dans les sociétés humaines, de la construction proposée ? (c) la théorie "politique" des conventions proposée dans l'ouvrage estelle assise sur des bases solides?

(a) Dans la postface de l'ouvrage, François Eymard-Duvernay signale " ...l'apport majeur de l'Economie des conventions à la théorie néoclassique..." (Eymard-Duvernay, p. 281). Il n'est pas besoin de faire une exégèse particulière de cette affirmation pour comprendre que s'il y a apport, c'est que l'Economie des conventions se situe à l'intérieur du cadre néoclassique. Soit. Mais, si tel est le cas, faudrait-il se résigner à l'abandon du caractère hétérodoxe que l'on prêtait jusque là au conventionnalisme? En fait, orthodoxie et hétérodoxie n'ont pas d'importance en soi. Ce qui importe avant tout, c'est la puissance des outils dont se dote telle ou telle école de pensée, ou tel ou tel paradigme. Si l'on revient à l'ouvrage, on ne peut que constater, ne serait-ce que par les références bibliographiques, que l'Economie des conventions n'a pas d'histoire ${ }^{2}$, et, n'ayant pas d'histoire, n'a pas eu d'autre projet d'intelligence du social que celui qui consiste à "psychologiser autrement" l'agent individuel. A cet égard, qu'il nous soit permis de rappeler que l'acte de naissance de l'Economie des conventions a été signé par des économistes, nombre d'entre eux administrateurs de l'INSEE, qui ont mis au premier plan de leurs préoccupations la construction et la signification des catégories statistiques, considérant par exemple que les statistiques du chômage " renvoient à la structure du marché du travail, à un mode de régulation, à des comportements sociaux et des modèles de représentation intégrés dans l'action des pouvoirs

\footnotetext{
2 Les ouvrages suivants ne sont en effet pas cités: R. Salais, N. Baverez et B. Reynaud, L'invention du chômage, 1986 ; A. Desrosières, L. Thévenot, Les catégories socioprofessionnelles, 1988. On peut également s'étonner de voir Alain Desrosières placé au rang de simple "applicateur" de l'économie des conventions aux conventions statistiques, dans l'encadré de la page 16. On s'étonnera aussi de l'absence de référence à R. Salais, E. Chatel et D. Rivaud-Danset (dir.), Institutions et conventions. La réflexivité de l'action économique, Paris, Editions de l'EHESS, 1998.
} 
publics. " ${ }^{3}$ Ce type de questionnements ouvrait la voie à une analyse des liens étroits entretenus entre catégories statistiques et catégories juridiques ; l'ouvrage de Salais, Baverez et Reynaud (1986) contenait des pistes prometteuses sur l'étude des catégories juridiques comme support de dispositifs informationnels, notamment statistiques, de la formulation des politiques économiques. Mais cette voie n'a pas été réellement suivie.

(b) Un trait remarquable des analyses rassemblées dans l'ouvrage réside dans un paradoxe: il pose explicitement que le problème central est la coordination à base de règles, mais l'associe à une typologie qui est à notre sens davantage fonctionnelle pour le modèle d'analyse, que substantielle: les règles-contrat, règles conventionnelles et règles-contraintes ne sont en effet jamais définies de manière un tant soit peu substantielle. Des illustrations anecdotiques (du genre : à quelle heure se passe une pause-café, ou dois-je m'habiller différemment selon les lieux où je me rends ?) ne sont guère éclairantes. Le paradoxe réside dans cet écart entre l'affirmation du caractère central des règles, et la légèreté des applications proposées, qui ne sont que de simples anecdotes, d'ailleurs revendiquées comme telles. Comment expliquer ce paradoxe ? Nous proposons trois hypothèses.

La première est que l'objet "règle" est finalement davantage une catégorie mineure, instrumentale, que le centre du projet de théorisation ${ }^{4}$. Ce projet tient à la modélisation des interactions entre agents, pensée en contraste avec cette référence constante qu'est la théorie des jeux, en vue de saisir les conditions épistémiques d'émergence des conventions. Or, la convention dont il est question n'a d'existence qu'immatérielle et cognitive : c'est "une forme d'accord non explicite" dont la prévalence n'est en rien rattachable "aux lois juridiques" (Batifoulier et de Larquier, p. 12). Cette orientation hors du juridique est à l'évidence légitime (bien que non conforme aux souhaits formulés par O. Favereau à de nombreuses occasions), notamment lorsqu'il s'agit de prolonger le programme keynésien d'analyse du phénomène conventionnel, qui se rattache essentiellement à la formation des croyances, et dont Favereau dégage les affinités avec la thèse de Lewis : formulation implicite, origine intraçable, absence de sanction juridiques, etc. (Favereau, p. 7). Toutefois, ce qui est contestable tient à l'extension de ce cadre au-delà de la formation des croyances, de manière à rendre du

3 R. Salais, N. Baverez et B. Reynaud, L'invention du chômage, 1986 - réédition PUF/Quadrige, 1999, p. 13.

${ }^{4}$ En somme, la règle est convoquée pour être mieux neutralisée par la convention. Sur ce point, voir les éclaircissements proposés par E. Serverin, "Propos croisés entre droit et économie sur la place de l'incertitude et de la confiance dans le contrat", Sociologie du travail, $\mathrm{n}^{\circ} 4,1996$, pp. 607-619. 
compte du fait que "l'activité économique [est] influencée par des règles de nature particulière, nommées "conventions" " (Batifoulier et de Larquier, p. 10). Le projet est là encore légitime, mais les modalités de son exécution posent problème, au regard de deux considérations: d'une part, l'ancrage des conventions dans un monde abstrait de coordination pure fondée sur des interactions exclusivement cognitives $^{5}$, et d'autre part des erreurs manifestes d'interprétation du monde réel. Il en va ainsi lorsque Batifoulier et de Larquier traitent des "conventions non juridiques", et prennent les exemples du code de la route, de l'heure des pauses-café et des tenues vestimentaires dans les entreprises. Il est surprenant de voir dans le code de la route une "convention non-juridique", exempte de sanctions légales, alors que les affaires d'infraction à ce code occupent un volume considérable de l'activité des services de police et des tribunaux; il est également surprenant de considérer que l'heure de la pause-café et la tenue vestimentaires sont en dehors du droit, alors que l'invocation de ces "conventions" comme usages est un registre très fréquemment mobilisé dans des conflits réglés par les conseils de prud'hommes ${ }^{6}$.

La deuxième hypothèse qu'il est raisonnable de formuler tient à la persistance d'une faible réflexivité - par rapport aux sciences sociales - de l'analyse économique des conventions, et de cette sorte de conventionnalisme défendu dans la troisième partie de l'ouvrage, qui se consacre à l'économie des règles interprétables. Là encore, le paradoxe est présent, cependant sous une forme inédite : l'affirmation que le rapport des règles aux comportements n'est pas épuisé par les modèles "stratégiques" conduit à placer l'accent sur la "coordination des représentations", lesquelles posent le problème de l'interprétation, traité sans qu'une théorie de l'interprétation soit exposée. Enorme découverte, en effet, que l'interprétabilité des règles... qui a été traité dans des milliers de pages en théorie du droit depuis 150 ans. De ces milliers de pages, seules quelques unes de Ronald Dworkin sont citées dans l'ouvrage. Et elles ne sont représentatives que d'un certain courant de pensée nord-américain centré sur la décision des juges de la Cour suprême des Etats-Unis dans les hard cases, ces cas judiciaires qui posent problème au regard des précédents existants. Là encore, la position des règles et de leur interprétation paraît être formulée de manière ad hoc dans

5 N. Postel est particulièrement explicite sur ce point dans son article (non cité) : "L'économie des conventions: une approche instrumentale de la rationalité individuelle ?”, Revue économique, vol. 49, n6, 1998, pp. 1473-1496.

${ }^{6}$ L'heure de la pause-café entre collègues de l'université n'est pas représentative de l'heure de la pause-café dans le monde réel (celui du salariat et de la fonction publique hors université). Sur la force légale des usages d'entreprise, voir la thèse de droit privé de Boris Bertin, L'usage face au droit étatique dans les relations de travail, Université de Saint-Etienne, 2001. 
un cadre théorique qui ne devrait tenir, in fine, que de la psychologie sociale : les règles dont parle l'ouvrage, ce ne sont au fond pas autre chose que des régularités de comportement, basées sur des représentations communes, transmises et entretenues par des mécanismes (non explicités) de socialisation. Ce ne sont pas des règles d'organisation des rapports économiques, sociaux ou politiques d'agents économiques-citoyens et sujets de droit7. Même si chacune de ces perspectives présente son intérêt intrinsèque, il est donc important de bien les distinguer.

Enfin, dernière hypothèse, l'absence de théorie substantielle des règles, et de leur interprétation, peut être imputée aux hésitations du conventionnalisme vis-à-vis des institutions. Là encore, nous avons une théorie des conventions sans institutions. Pourtant, un non contributeur à l'ouvrage, Hervé Defalvard ${ }^{8}$ a diagnostiqué une convergence entre les trois courants de l'Economie des conventions, caractérisés par leur entrée dans le champ de l'institutionnel : les normes sociales (branche Orléan) ; le choix social (branche Salais) ; l'interdépendance des niveaux de décision (branche Favereau) ${ }^{9}$. Cette convergence touche à la théorisation de l'action individuelle en société, dont Defalvard estime qu'elle est désormais communément placée sur le registre de l'analyse de la "coordination des actions situées dans des espaces institutionnels " (Defalvard, p. 9). Cet auteur se propose d'étudier la part des institutions dans les conduites individuelles, en développant une analyse qualifiée d'institutionnaliste de la coordination sociale... en termes de jeux. Cette méthode revient à formaliser les institutions à un niveau macro-institutionnel, préalable à la formalisation au niveau microinstitutionnel; le formalisme retenu s'appuie sur l'idée que les institutions n'ont d'existence que symbolique, considérant que "n'ayant aucune existence", elles n'ont "qu'une essence " (Defalvard, p.16).

De notre point de vue, cette opération de désubstantialisation (et de dématérialisation) des institutions va à rebours du projet d'intelligence pratique de la coordination située; en effet, à ce formalisme de l'action située peut être opposé le réalisme

\footnotetext{
${ }^{7}$ Ce qui serait intéressant, ce serait de voir se développer des travaux sur deux cents ans d'interprétation de l'article 1382 du Code civil, formidable metteur en scène de transferts de richesses entre membres d'une collectivité humaine... ou sur les comportements des entreprises d'assurance orientés par cette règle du Code civil...

8 H. Defalvard, "L'Economie des Conventions à l'école des institutions", Document de travail du Centre d'études de l'emploi, $\mathrm{n}^{\circ} 2$, juillet 2000.

9 Signalons que les analyses de Postema de la coordination et des conventions au regard du droit sont complètement méconnues dans le champ de l'économie des conventions : voir G.J. Postema, "Coordination and Convention at the Foundations of Law”, Journal of Legal Studies, vol. XI, pp. 165-203, 1982.
} 
pragmatique des travaux d'Anne-Lise Aucouturier sur la mise en œuvre des dispositifs de l'action publique (en l'occurrence l'aide aux chômeurs créateurs d'entreprise ${ }^{10}$ ), ou d'Elisabeth Chatel sur l'évaluation éducative ${ }^{11}$, qui relèvent d'une même approche conventionnaliste de l'action des institutions dans le monde réel.

(c) L'Economie des conventions se veut donc désormais "politique ", ainsi que l'indique la postface de François EymardDuvernay. On ne peut que se réjouir de la réapparition cet adjectif, sans doute trop longtemps enterré. Mais, curieusement, les auteurs ne s'appuient pas sur les acquis, pourtant considérables, de ce que l'ont serait tenté d'appeler la "première Economie des conventions" dans ce domaine. En effet, des travaux pionniers comme ceux d'Alain Desrosières et Laurent Thévenot avaient, dès 1988, posé les premiers jalons d'une économie politique de la coordination. Selon ces auteurs, "une classification socioprofessionnelle ne peut être traitée comme n'importe quel outil statistique dont il s'agirait d'expliquer le fonctionnement. Elle est étroitement liée aux opérations de représentation d'une société: représentation statistique, certes, obéissant à certaines contraintes techniques; représentation politique, ensuite, puisque l'on parle aujourd'hui couramment des "socioprofessionnels" pour des personnes ayant un mandat comparable à celui d'autres élus ; représentation cognitive ordinaire, enfin, dont chacun dispose pour se repérer et faire des rapprochements dans la vie en société, et qui a rapport avec la profession ou le milieu social." (p. 7) Il s'agit donc pour eux de replacer en contexte (en accordant notamment un rôle central à l'Etat) les représentations individuelles. Ce replacement est d'ailleurs productif, puisque, pour caricaturer, il leur permet d'apporter des réponses là où les auteurs de l'ouvrage collectif en sont encore à se poser des questions, quinze ans après. En particulier, Desrosières et Thévenot montrent que les individus sont capables de se repérer dans l'univers social, et ce quelle que soit leur position dans cet univers.

\footnotetext{
10 A.-L. Aucouturier, Evaluation des politiques d'emploi et action publique, thèse de doctorat, Université de Paris X-Nanterre, 1998 ; A.-L. Aucouturier et C. Didry, Des politiques de l'emploi à la pluralité des formes juridiques de la relation de travail, analyse socio-économique de deux expériences : l'aide aux chômeurs créateurs d'entreprise et le rôle du Comité d'Entreprise dans le contrôle des plans sociaux, Rapport pour le Commissariat Général du Plan, juin 1997.

${ }^{11} \mathrm{E}$. Chatel, Une analyse économique de l'action éducative : évaluation et apprentissage dans les lycées, thèse de doctorat en sciences économiques, Université de Paris X, 1996 ; E. Chatel, Comment évaluer l'éducation. Pour une théorie sociale de l'action éducative, Paris, Delachaux et Niestlé, 2001.
} 
Mais ce premier résultat d'importance de ce qui allait devenir $1^{\prime} E$ Economie des conventions a été oublié ${ }^{12}$. A sa place, nos auteurs préfèrent garder les prémisses de la théorie néo-classique d'un "agent" désocialisé, sans repères ni mémoire, qui ne connaît pas la société dans laquelle il vit. Ainsi, le livre affirme que le "projet initial " de l'Economie des conventions est de "fonder une économie politique sur la base d'une théorie renouvelée $d u$ choix rationnel" (p. 218, nous soulignons) ${ }^{13}$. A ce cerveau, on adjoint des "principes de justice" à l'origine non explicitée. Comment la coordination entre de tels êtres se fait-elle ? La réponse est donnée par Olivier Biencourt, Camille Chaserant et Antoine Rebérioux dans un paragraphe intitulé Le programme conventionnaliste comme économie politique (Ch. 6). Selon eux, "la coopération repose non pas tant sur une régulation des comportements - opérée par le suivi des règles conventionnelles - que sur une régulation des représentations. Plus précisément, l'action collective présuppose une harmonisation des façons de penser et de juger. (...). Il importe donc davantage d'aligner les représentations que les actions elles-mêmes; il s'agit sans doute là du coeur du message de l'Economie des conventions. " (p. 211, nous soulignons). Du constat de l'efficacité de la stabilité des anticipations croisées dans des contextes (objet de la première partie du livre), nos auteurs glissent donc à la revendication normative de la nécessité souhaitable de normaliser les représentations. On tremble devant les moyens qu'il faudra employer pour " réguler " ou "aligner" les représentations, surtout s'il s'agit d'en faire un programme politique...

La postface de François Eymard-Duvernay propose justement de développer une "théorie politique " de l'Economie des conventions. Cependant, pour lui, l'Economie des conventions ne trouve pas sa source dans la théorie du choix rationnel mais dans "les développements modernes des sciences cognitives et de la philosophie " (p. 280) ${ }^{14}$. L'article présente ensuite un dialogue entre plusieurs approches, à commencer par celles de Rawls et de Bowles et Gintis. De cette lecture, François Eymard-Duvernay conclut que "deux points majeurs " ressortent de la relecture de Rawls par les radicaux américains: "le rôle central joué dans les économies capitalistes par les grandes firmes" et "l'importance donnée aux processus d'apprentissage ". Or, selon lui, "comme l'a montré le chapitre $8, l^{\prime}$ Economie des conventions tente de maintenir ces deux

12 Et ce malgré l'ouvrage de François Dosse qui consacre trois chapitres aux origines de l'Economie des conventions (voir F. Dosse, L'empire du sens, La Découverte, 1995, Sixième Partie, Les conventions : une troisième voie ?). Mais cet ouvrage n'est pas cité dans la bibliographie.

13 Ce "projet initial " n'est donc pas celui des travaux initiaux de Desrosières et Thévenot, ni celui de Salais, Baverez et Reynaud.

14 Ce qui est cohérent avec le but, effectivement "urgent", de "construire une alternative institutionnaliste à la théorie standard " (id.). 
lignes de recherche" (p. 288). Il s'agit alors de "compléter" cette approche par les apports de l'Economie des conventions, notamment ceux de Boltanski et Thévenot. François Eymard-Duvernay présente alors leurs thèses en miroir de celles de Rawls, en insistant sur leur différence majeure, à savoir la présence d'une pluralité des principes de justice chez les premiers. Comme son but est de construire une théorie de 1' "action publique ", il indique alors que du modèle des Economies de la Grandeur, on pourrait inférer " qu'il y a de l'Etat implanté dans le marché et dans les entreprises " (p. 292), c'est-àdire partout... alors qu'on peut penser que l'Etat est justement le grand absent de la construction théorique de Boltanski et Thévenot. Par contre, l'appui sur Boltanski et Thévenot lui permet d'indiquer que "le marché (...) ne peut être une forme de coordination générale, compatible avec toutes les conceptions du bien " (p. 293), ce qui est en effet un résultat crucial.

Mais ce n'est de toutes façons pas dans la direction de l'Etat que se tourne Eymard-Duvernay pour analyser "le passage du général au particulier, des principes de justice à l'action" (p. 294). Suivant les radicaux américains, il préfère en effet se tourner vers les " institutions intermédiaires ". Celles-ci permettent de résoudre la tension entre le "registre du "bien", dans lequel l'individu poursuit intensément les fins qu'il jugent bonnes " et " un registre "politique" dans lequel il comprend et intègre dans son comportement la pluralité des valeurs" (p. 296). Mais quelles sont ces institutions intermédiaires ? Pour François Eymard-Duvernay, "une solution à ce problème consiste à construire des "communautés", fondées sur la proximité, et dans lesquelles les valeurs sont homogènes" (p. 296, nous qui soulignons). Certes, il ne s'agit là que d'une solution, mais sa proximité avec la "régulation des représentations ", autre solution proposée plus haut au problème de la coordination est frappante. Dans les deux cas, la question de la coordination entre des êtres peu ou pas institués se "résout" dans la négation des différences interindividuelles, les représentations ou valeurs des agents devant etre strictement identiques pour que la coordination soit possible.

La vertu principale de cet ouvrage aura donc été, selon nous, de rendre explicite pour la première fois cette version de l'Economie des conventions. Celle-ci, comme on le voit, s'est donnée pour tâche de réintroduire dans l'analyse économique standard des éléments qui lui sont pour l'instant étrangers, comme les entreprises, voire les systèmes de valeurs et les relations politiques, afin d'expliquer les phénomènes de coordination. Mais est-il raisonnable de vouloir atteindre de tels buts en gardant un cahier des charges aussi contraignant que celui que l'économie standard s'est imposée à ellemême? 